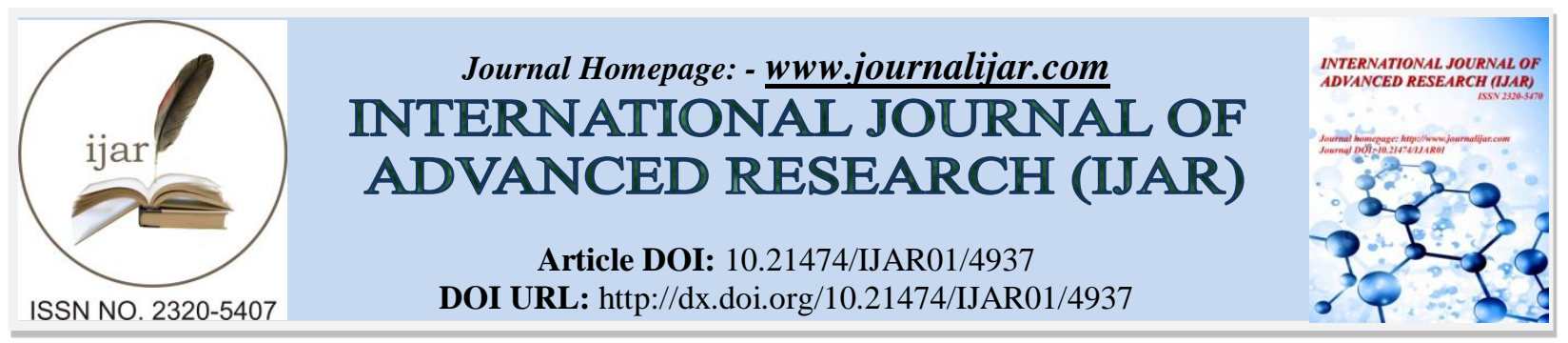

RESEARCH ARTICLE

\title{
FONCTIONNEMENT HYDRODYNAMIQUE DE LA LAGUNE DE OUALIDIA (MAROC) AVANT L'AMENAGEMENT DE LA SOUILLE.
}

Hilmi Karim, Makaoui Ahmed, Ettahiri Omar, Idrissi Mohammed, Larissi Jamila, Abdellaoui Benyounes, El Ouehabi Zineb and et Orbi Abdellatif.

Institut National de Recherche Halieutique Casablanca, Maroc.

\section{Manuscript Info}

[.........................

Manuscript History

Received: 24 May 2017

Final Accepted: 26 June 2017

Published: July 2017

Key words:-

lagoon, Morocco, current, tide, temperature/salinity, underground freshwater seepage, renewal time.

\section{Abstract}

The hydrodynamic functionning of Oualidia lagoon, located on the Atlantic coast of Morocco $\left(32^{\circ} 45^{\prime} \mathrm{N}-9^{\circ} 30^{\prime} \mathrm{W}\right)$, is investigated by tide, current, temperature-salinity observations during summer /autumn 2005 and hydrodynamic 2D model, before the installation of the sediment trap located upstream of the lagoon. The observed tide is semi-diurnal tide (mainly M2) of $0.97 \mathrm{~m}$ amplitude, asymetric and, propagating outside the inlets to upstream of the lagoon (artificial dam), its phase's delay is around of $3 \mathrm{~h}$. The tidal currents are very intense $\left(>1 \mathrm{~m} . \mathrm{s}^{-1}\right)$ near the inlets of the lagoon with flood's dominance and are less intense $\left(<0.5 \mathrm{~m} . \mathrm{s}^{-1}\right)$ upstream of the lagoon. Water temperature and salinity observed at different stations located in the main channel indicate a clear influence of marine water downstream and the salinity is reduced upstream of the lagoon, due to several underground freshwater seepages in the lagoon. The local renewal time (LRT) is varying between 1 day near the inlets of the lagoon to around 30 days at its artificial dam. The integral renewal time (IRT) of Oualidia lagoon is 15 days in mean (Koutitonsky et al., 2007).

Copy Right, IJAR, 2017,. All rights reserved.

\section{Introduction:-}

La lagune de Oualidia $\left(32^{\circ} 45^{\prime} \mathrm{N}-9^{\circ} 30^{\prime} \mathrm{O}\right)$, située sur la façade Atlantique marocaine entre El Jadida et Safi (figure 1), est l'un des sites littoraux nationaux où l'ostréiculture s'est développée de manière artisanale depuis les années 1950. Elle s'étend parallèlement à la côte sur une distance approximative de $7.5 \mathrm{~km}$ de long et de $0.5 \mathrm{~km}$ de large. Installée à la faveur d'une dépression interdunaire, sa morphologie est constituée de: i) de deux passes d'entrée de dimensions inégales à travers le cordon littoral constitué de dunes consolidées: une passe principale permanente ( $150 \mathrm{~m}$ de large environ et $3 \mathrm{~m}$ de profondeur en moyenne) et une passe secondaire (50 $\mathrm{m}$ de large environ et $1 \mathrm{~m}$ de profondeur en moyenne) active en périodes de marées de vives eaux; ces deux passes permettent les échanges avec la mer et le renouvellement des eaux de la lagune; ii) un delta de marée interne ou «sablière» dont sa morphologie est variable dans l'espace et dans le temps (Carruesco, 1989) et soumis en permanence à l'action conjuguée de vagues/houles et de courants de marée (Hilmi et al., 2005 a et b; Koutitonsky et al., 2006a, 2007) ; iii) d'un chenal principal sinueux ( $5.4 \mathrm{~km}$ de long sur près de $0.4 \mathrm{~km}$ de large environ) et de plusieurs chenaux secondaires, la profondeur moyenne du chenal principal dépasse les $4 \mathrm{~m}$ à certains endroits et près de la moitié (53\%) de la surface de la lagune se découvre à marée basse; la plupart des parcs ostréicoles sont situés dans cette zone centrale de la lagune et iv) dans la partie amont de la lagune, une digue amont sépare la digue artificielle aval des salines exploitées de manière artisanale en saisons d'été (figure 1); suite à des problèmes de confinement observés en amont 
de cette lagune (GEME, 2002, 2003 et 2004), les pouvoirs publics ont décidé d'aménager une souille ou «piège à sédiment» entre les digues aval et amont qui est fonctionnelle depuis 2011.

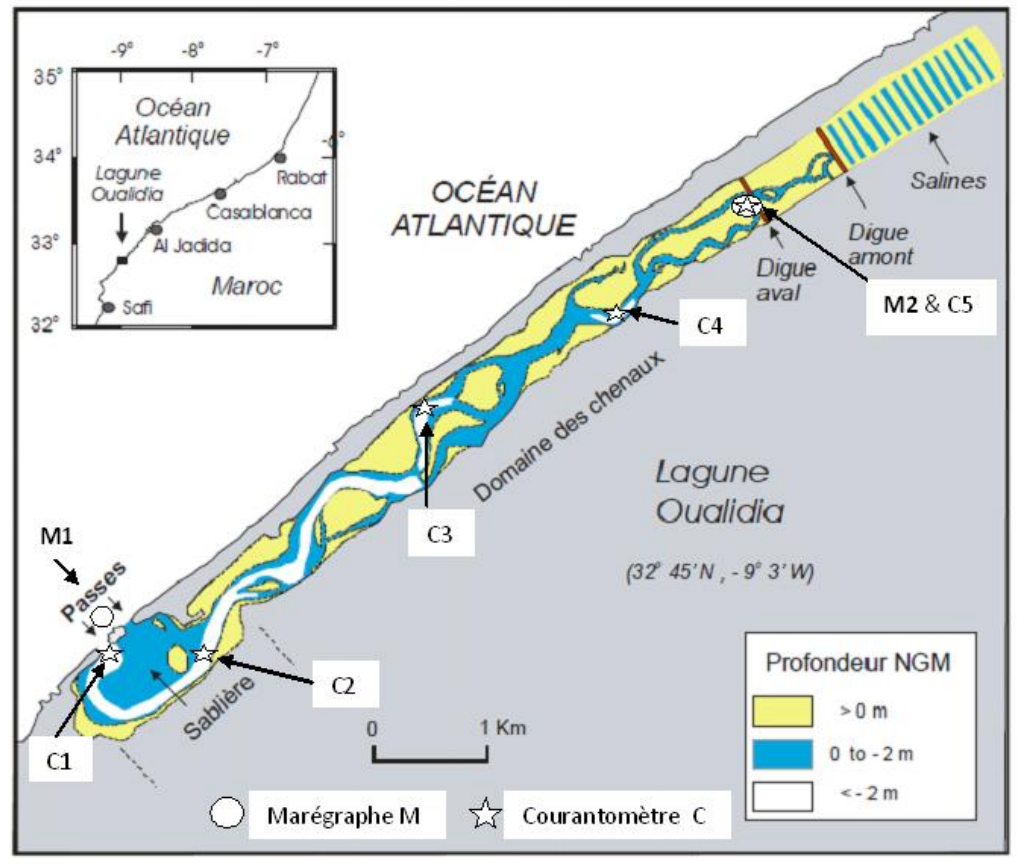

Figure 1:- Situation géographique de la lagune de Oualidia $\left(32^{\circ} 45^{\prime} \mathrm{N}-9^{\circ} 30^{\prime} \mathrm{O}\right)$ et campagne de mesures effectuée par l'INRH en saisons d'été et d'automne 2005 (Carte adaptée de Koutistonsky et al, 2006a).

Jusqu'en 2005, la digue aval artificielle était quasiment «fermée» présentant une brèche de $10 \mathrm{~m}^{2}$ environ communiquant avec la lagune, limitant ainsi le domaine étudié de la lagune entre les passes d'entrée et cette digue aval (figure 1). De par son importance socio-économique pour la région, cette lagune a fait l'objet de diverses études visant les aspects sédimentologiques, géologiques et biogéochimiques (Bidet, 1981; Carruesco, 1989; Damsiri et al., 2015; El Khalidi et al., 2011; Maanan et al., 2014; Sarf, 1999; Zourarah, 2002...), biologiques (Bennouna, 1999, 2002; Ouledessaïb,1997, ...); environnementaux et liés à la pollution (Cheggour et al., 1999; El Attar, 1998; Hassou et al., 2014; Hennani et al., 2014; Lakhdar et al., 2005; Idardare et al., 2014; ...); les aspects liés à l'aquaculture (Berraho; 1998; Rharbi et al., 2001, 2003; INRH, 2002; Kamara et al., 2005, 2008; Shafee et Sabatie 1986,...). Les études portant sur les aspects hydrodynamiques sont plus récentes mais peu nombreuses. On citera notamment les travaux de Hilmi et al., (2005a et b, 2006, 2009); Koutitonsky et al., (2006, 2007, 2012); Orbi et al., (2008), Zourarah et al., 2012). L'objectif de cette étude est de décrire, d'une part, les paramètres régissant la circulation et l'hydrologie de la lagune, à savoir la marée, le courant, la température et la salinité à plusieurs stations de mesures effectuées durant l'été et l'automne 2005. La particularité de cette campagne de mesures réalisée par l'INRH en 2005 est qu'elle a porté sur environ six mois d'observation à plusieurs stations de mesures, réparties de l'aval (passes d'entrée) vers l'amont (digue aval artificielle) de la lagune. Il s'agit de contribuer, d'autre part, à la compréhension du fonctionnement hydrodynamique de cette lagune, à partir de résultats portant sur la modélisation hydrodynamique 2D menée aussi bien en 2005 (Hilmi et al., 2005b) qu'après 2005, notamment par Koustitonsky et al. (2006a, 2007) avant l'aménagement de la souille.

\section{Climat de la zone d'étude:-}

\section{Température de l'air:-}

D'après Carruesco (1989), les températures atmosphériques à Oualidia varient en moyenne entre $21^{\circ} \mathrm{C}$ à $25^{\circ} \mathrm{C}$ en été et entre $13^{\circ} \mathrm{C}$ à $16^{\circ} \mathrm{C}$ en hiver, avec une moyenne annuelle de $18^{\circ} \mathrm{C}$. De très fortes températures extrêmes pouvant atteindre $40^{\circ} \mathrm{C}$ peuvent être observées en été, lorsque souffle le vent dit «Chergui» (ENE), originaire des «dépressions sahariennes» qui contribue à augmenter le phénomène d'évaporation. Les moyennes annuelles de ce dernier paramètre sont estimées entre $1300 \mathrm{~mm}$ et $1500 \mathrm{~mm}$ (Ferre et Ruhard, 1975). L'humidité de l'air joue aussi un rôle important dans la frange littorale. Les maximas sont voisins de100\% lors des journées à brouillards matinaux et les minimas absolus sont atteints les jours de «Chergui » (moins de 10\%) (Carruesco, 1989). 


\section{Vent:-}

Une excellente synthèse sur les vagues/houles et les vents au large de Oualidia et à proximité de la lagune a été réalisée par Koutistonsly et al. (2006a). Selon ces derniers auteurs, les vents et la pression atmosphérique sont deux facteurs qui peuvent faire varier les niveaux d'eau et les courants aux passes d'entrée de la lagune de Oualidia. Les vents sont aussi responsables de la génération des vagues au large des côtes. Ces vagues se propagent vers la côte sous forme de vagues de tempête ou, en l'absence de vent, sous forme de houle (Koutistonsly et al. , 2006a). Les statistiques des vents du large annuels et saisonniers au large de Oualidia indiquent que i) les vents annuels moyens ont des vitesses variant entre 4 et $9 \mathrm{~m} . \mathrm{s}^{-1}$, avec des directions prédominantes du nord-est et du nord ; ii) les plus forts vents sont observés durant les mois de décembre à février, atteignant des vitesses au delà de $30 \mathrm{~m} . \mathrm{s}^{-1}$, surtout en provenance de l'ouest et du nord-ouest et iii) les plus faibles vents se manifestent durant l'été et soufflent surtout du nord et du nord-est (Koutitonsky et al., 2006a). Pour les vents côtiers, il n'existe pas de longues séries temporelles de vent sur la lagune de Oualidia et la plupart des études font référence aux travaux de Carruesco (1989). Les directions prédominantes des vents de mars à août sont celles du nord-est et du nord. Durant les autres mois de l'année, les vents proviennent également de ces directions mais avec des occurrences plus fréquentes du sud-ouest, surtout durant l'hiver (décembre à février) (Carruesco, 1989; Koutistonsly et al.. 2006a)

\section{Vagues et houles:-}

Selon Koutitonsky et al. (2006a et 2007), les plus grandes vagues proviennent des secteurs nord, nord-ouest et ouest. Plus spécifiquement, les plus hautes vagues (10-11m) proviennent de l'ouest et les plus longues vagues (12-13 secondes) proviennent du nord-ouest et du nord. Les vagues qui progressent du large vers la lagune de Oualidia sont des vagues d'eaux profondes et leur direction n'est pas affectée par la friction du fond. Lorsque ces vagues s'approchent des côtes, elles commencent à subir l'influence du fond et à être réfractées. Les vagues qui progressent du large vers la lagune de Oualidia subiront une réfraction par les hauts fonds près des côtes à mesure qu'elles se rapprochent de la passe d'entrée de la lagune et déferlent. La vague qui a déferlé juste à l'extérieur de la passe d'entrée de la lagune Oualidia va se reformer et, par haute mer, pénétrer dans la lagune par la passe et se propager sur les hauts fonds de la sablière où elle déferlera à nouveau. Des vagues de faible hauteur pénètrent dans la lagune la plupart du temps. Les vagues moyennes se propagent jusqu'à la rive sud de la sablière, en perdant progressivement leur amplitude en déferlant sur les hauts fonds de la sablière. Les vagues extrêmes, y compris celles du nord-est, pénètrent dans la lagune avec des hauteurs légèrement plus grandes. Comme les vagues moyennes, elles déferlent en dehors de la lagune et se reconstituent dans la région de la passe d'entrée. Lorsqu'elles atteignent les hauts fonds de la sablière, elles déferlent à nouveau. Les vagues en provenance de l'ouest auront un impact plus prononcé sur la partie est de la sablière. Toutes les vagues atteignent le côté sud du chenal principal qui longe la rive sud de la lagune. Les courants orbitaux associés aux vagues moyennes et extrêmes dans la lagune affichent la plupart du temps des vitesses qui dépassent largement $0.3 \mathrm{~m} \cdot \mathrm{s}^{-1}$ (Koutitonsky et al., 2007). Selon ces auteurs, les événements de grandes vagues du large vont contribuer de façon significative à la dynamique de la sablière et à son remaniement dans le temps. Ces fortes vitesses vont donc contribuer au transport de sédiments non-cohésifs dans la région de la sablière de la lagune (Koutitonsky et al., 2007).

\section{Matériel et methodes:-}

Afin d'étudier la propagation de la marée au sein de la lagune, deux marégraphes de marque «Aanderaa» ont été installés en 2005 par l'Institut National de Recherche Halieutique (INRH) à l'extérieur et au sein de la lagune: le premier à l'extérieur de la lagune à la station M1 sur une période de six mois environ (du 24 juillet au 01 décembre 2005) et le deuxième à proximité de la digue aval artificielle à la station M2 sur un mois environ (du 24 juillet au 18 aôut 2005) (figure 1). L'asymétrie de la marée au sein de la lagune a étudiée par modèle hydrodynamique 2D (Hilmi et al., 2005b; Koutitonsky et al., 2007). Pour l'étude du courant, cinq courantomètres de marque «Aanderaa» ont été installés du 24 juillet au 22 août 2005 à cinq stations de mesures réparties le long du chenal principal de la lagune, de l'aval (en retrait de la passe d'entrée principale) à l'amont de la lagune (digue aval artificielle). Enregistrés à un pas temporel de $20 \mathrm{mn}$, les paramètres observés à chacune de ces stations de mesures sont: la marée (m) pour les marégraphes M1 et M2, la température $\left({ }^{\circ} \mathrm{C}\right)$ et la salinité $(\mathrm{psu})$ de l'eau, l'intensité $\left(\mathrm{m} . \mathrm{s}^{-1}\right)$ et la direction du courant (en degrés) pour les courantomètres $\mathrm{C} 1$ à $\mathrm{C} 5$. Les séries temporelles du niveau d'eau (stations M1 et M2) et des composantes du courant (stations C1 à C5) ont été, au préalable, lissées afin d'en éliminer les fluctuations de périodes inférieures à $3 \mathrm{~h}$ (Godin, 1972). Les séries temporelles du niveau d'eau ont été soumises à l'analyse harmonique (Pawlowicz et al., 2002). Cette analyse permet d'extraire la variance de la série et de l'assigner à diverses composantes harmoniques de la marée. Ces mêmes séries (niveau d'eau et courant) furent également soumises à l'analyse spectrale (Bendat et Piersol, 1986), afin d'étudier la répartition de l'énergie cinétique dans les basses et hautes fréquences. 


\section{Résultats et Discussion:- \\ Marée et asymétrie:-}

La figure 2 représente les mesures de la marée observées à l'extérieur de la passe d'entrée principale de la lagune (station M1) du 24 juillet au 01 décembre 2005 (figure 1). Les résultats de l'analyse harmonique (Hilmi et al., 2006) du niveau d'eau à la station M1 (figure 1) sont présentés au tableau 1. Le nom de l'harmonique, sa fréquence (en cycles par heure ou $\mathrm{cph}$ ), son amplitude (en $\mathrm{m}$ ) et sa phase (en degrés) avec leurs intervalles de confiance de 95\% respectifs y sont mentionnés. Ces résultats indiquent que l'amplitude de la marée à l'entrée de la lagune de Oualidia est dominée par l'harmonique semi-diurne M2 (amplitude $0.97 \mathrm{~m}$ ), suivie par les amplitudes des harmoniques S2 (amplitude $0.35 \mathrm{~m}$ ) et $\mathrm{N} 2$ (amplitude $0.20 \mathrm{~m}$ ). Leurs phases respectives sont de 49 degrés, 67 degrés et 30 degrés (tableau 1). Concernant les harmoniques diurnes, ce sont les harmoniques K1 (amplitude $0.07 \mathrm{~m}$ ) et O1 (amplitude $0.06 \mathrm{~m}$ ) qui suivent avec des amplitudes plus faibles (tableau 1). Le rapport «F ou Form Number » (Pugh, 1987) qui est égal au rapport d'amplitude des ondes diurnes $\mathrm{K} 1$ et $\mathrm{O} 1$ à celui des ondes semi diurnes M2 et S2 est inférieur à 0,25 et, par conséquent, c'est l'onde semi-diurne M2 (de période 12,42h) qui domine dans la lagune de Oualidia (tableau 1). Ces résultats sont compatibles avec les travaux de Fanul et al., 1997; Hilmi et al., 2005 a et b; Orbi et al., 1998; Koutitonsky et al.; 2006a,... qui dénotent que la marée dominante au Maroc (façades atlantique et méditerranée) est de type semi diurne. L'analyse harmonique dénote également la présence de sous harmoniques (M3, M4, M6, ...) de la composante M2 (tableau 1) qui sont non négligeables et causées par l'interaction non linéaire de cette onde de marée M2 avec les faibles profondeurs de la lagune et la forte courbure et morphologie du chenal principal (Friedrichs and Madsen, 1982; Dronkers, 1998; Blanton et al., 2002 cités par Koutitonsky et al., 2007).

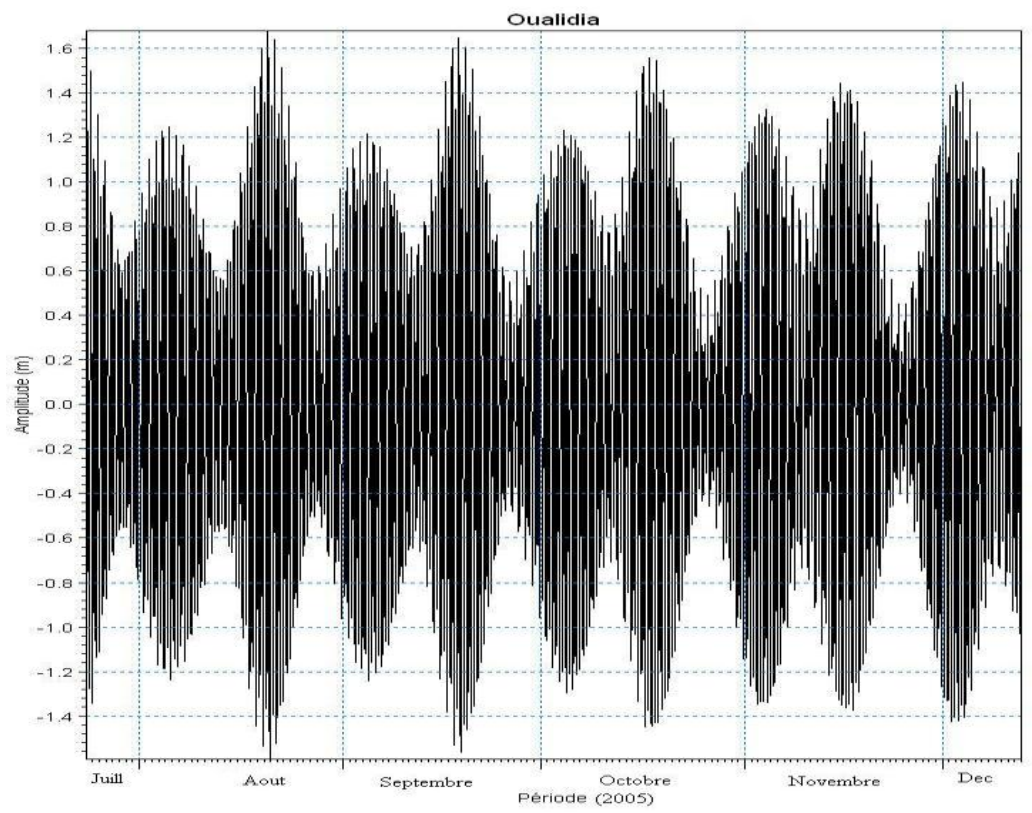

Figure 2:- Marée observée à l'entrée de la passe d'entrée principale (station M1) de la lagune de Oualidia sur la période de juillet à décembre 2015 (Source INRH- Hilmi et al., 2006).

En se basant sur une bathymétrie de la lagune réalisée avant 2005, la propagation de la marée au sein de la lagune de Oualidia fut étudiée par modèle hydrodynamique 2D (Hilmi et al., 2005b). De manière plus précise et en se basant sur une bathymétrie de la lagune plus 
Tableau 1:- Analyse hamonique du niveau d'eau observé à l'extérieur de la passe d'entrée (station M1) sur la période juillet à décembre 2005 incluant le nom de l'harmonique, sa fréquence (en cph), son amplitude (en m) et sa phase (en degrés) avec leurs intervalles de confiance de 95\% respectifs (d'après Hilmi et al., 2006):

\begin{tabular}{|c|c|c|c|c|c|}
\hline $\begin{array}{l}\text { Composante } \\
\text { Harmonique }\end{array}$ & Fréquence (cph) & $\begin{array}{c}\text { Amplitude } \\
\text { (m) }\end{array}$ & $\pm 95 \%$ & $\begin{array}{c}\text { Phase GMT } \\
\text { (Deg) }\end{array}$ & $\pm 95 \%$ \\
\hline MM & 0.0015122 & 0.0163 & 0.035 & 63.01 & 145.77 \\
\hline MSF & 0.0028219 & 0.014 & 0.032 & 247.43 & 153.34 \\
\hline ALP1 & 0.0343966 & 0.0028 & 0.002 & 172.83 & 48.15 \\
\hline 2Q1 & 0.0357064 & 0.0075 & 0.004 & 199.57 & 32.17 \\
\hline Q1 & 0.0372185 & 0.0227 & 0.004 & 245.58 & 9.82 \\
\hline O1 & 0.0387307 & 0.0605 & 0.004 & 302.09 & 3.84 \\
\hline NO1 & 0.0402686 & 0.003 & 0.002 & 28.23 & 41.89 \\
\hline P1 & 0.0415526 & 0.0226 & 0.005 & 59.50 & 13.41 \\
\hline K1 & 0.0417807 & 0.0684 & 0.004 & 52.43 & 3.67 \\
\hline $\mathrm{J} 1$ & 0.0432929 & 0.0030 & 0.002 & 147.64 & 47.88 \\
\hline OO1 & 0.0448308 & 0.0014 & 0.002 & 155.86 & 62.68 \\
\hline UPS1 & 0.046343 & 0.0013 & 0.001 & 7.12 & 79.14 \\
\hline EPS2 & 0.0761773 & 0.0072 & 0.01 & 338.58 & 88.05 \\
\hline MU2 & 0.0776895 & 0.0388 & 0.015 & 25.38 & 21.30 \\
\hline $\mathrm{N} 2$ & 0.0789992 & 0.2079 & 0.014 & 29.47 & 4.34 \\
\hline M2 & 0.0805114 & 0.9732 & 0.016 & 48.57 & 0.73 \\
\hline L2 & 0.0820236 & 0.0119 & 0.01 & 74.94 & 55.08 \\
\hline S2 & 0.0833333 & 0.3526 & 0.015 & 66.88 & 2.17 \\
\hline $\mathrm{K} 2$ & 0.0835615 & 0.0960 & 0.011 & 89.28 & 7.16 \\
\hline ETA2 & 0.0850736 & 0.0082 & 0.007 & 147.82 & 62.85 \\
\hline MO3 & 0.1192421 & 0.0006 & 0.001 & 290.61 & 114.81 \\
\hline M3 & 0.1207671 & 0.0033 & 0.001 & 230.41 & 24.37 \\
\hline MK3 & 0.1222921 & 0.0009 & 0.001 & 334.3 & 80.68 \\
\hline SK3 & 0.1251141 & 0.0010 & 0.001 & 59.86 & 62.2 \\
\hline MN4 & 0.1595106 & 0.0068 & 0.002 & 93.18 & 11.59 \\
\hline M4 & 0.1610228 & 0.0159 & 0.002 & 148.82 & 5.84 \\
\hline SN4 & 0.1623326 & 0.0020 & 0.001 & 212.05 & 43.45 \\
\hline MS4 & 0.1638447 & 0.0092 & 0.001 & 231.13 & 9.26 \\
\hline S4 & 0.1666667 & 0.0026 & 0.001 & 282.49 & 30.29 \\
\hline $2 \mathrm{MK5}$ & 0.2028035 & 0.0007 & 0.001 & 261.52 & 99.46 \\
\hline 2SK5 & 0.2084474 & 0.0006 & 0.001 & 61.66 & 107.73 \\
\hline 2MN6 & 0.2400221 & 0.0006 & 0.001 & 22.77 & 128.79 \\
\hline M6 & 0.2415342 & 0.0014 & 0.001 & 83.37 & 48.88 \\
\hline 2MS6 & 0.2443561 & 0.0014 & 0.001 & 141.64 & 51.45 \\
\hline 2SM6 & 0.2471781 & 0.0007 & 0.001 & 308.32 & 104.23 \\
\hline $3 \mathrm{MK} 7$ & 0.2833149 & 0.0014 & 0.001 & 49.74 & 45.16 \\
\hline M8 & 0.3220456 & 0.0004 & 0.001 & 38.15 & 170.07 \\
\hline
\end{tabular}

actualisée en 2006 et en utilisant les observations de la marée mesurée par l'INRH en 2005 à l'entrée de la passe principale de la lagune de Oualidia (station M1 figure 1), Koutitonsky et al. (2007) ont actualisé cette étude par modèle hydrodynamique 2D. La figure $3 \mathrm{a}$ et $\mathrm{b}$ présente ainsi les amplitudes et les phases de l'onde M2. Comme précisé au tableau 1, l'amplitude de l'harmonique M2 est de $0.97 \mathrm{~m}$ à l'extérieur de la lagune en océan Atlantique. $\mathrm{Au}$ fur et à mesure que l'onde M2 se propage dans la lagune, elle diminue d'amplitude à cause de la friction sur le fond et sur les zones intertidale et subit un retard de phase. Selon Koutistonsky et al. (2007), son amplitude subit une légère diminution au $\mathrm{km} 4$ de la lagune $(0.7 \mathrm{~m})$ puis elle est réduite à $0.3 \mathrm{~m}$ au niveau de la digue aval artificielle (Figure 3a) et, en calculant son retard de phase entre la passe d'entrée principale de la lagune à sa digue aval artificielle, l'amplitude de l'harmonique M2 met environ 3 heures pour progresser (figure $3 \mathrm{~b}$ ). Ces résultats sont conformes avec ceux de Hilmi et al. (2006), en étudiant le retard de phase entre les deux stations marégraphiques lors de la campagne de mesures effectuée en 2005. La faible atténuation de l'amplitude et le faible retard de phase 
suggèrent que la lagune de Oualidia est une lagune «coulante», c'est-à-dire que la marée se propage librement, par opposition à une lagune «étouffée » comme celle de Nador située en mer Méditerranée marocaine $\left(35^{\circ} 12^{\prime} \mathrm{N}\right.$ $02^{\circ} 51^{\prime} \mathrm{O}$ ) par exemple où la marée avant 2011 perdait la totalité de son amplitude à la passe d'entrée et où le retard de phase augmente à près de trois heures sous la configuration de son ancienne passe (Hilmi et al., 2005c; Koutitonsky et al., 2006b). L'asymétrie de la marée au sein de la lagune de Oualidia a été aussi étudiée par modèle 2D de manière plus précise par Koutitonsky et al. (2007) (figures $3 \mathrm{c}$ et d). Elle est caractérisée par le rapport des amplitudes des harmoniques $\left(\mathrm{A}_{\mathrm{M} 4} / \mathrm{A}_{\mathrm{M} 2}\right)$ et par le calcul de la phase relative entre les harmoniques $\mathrm{M} 2$ et M4 des niveaux (soit $\Delta \theta=2 \theta_{\mathrm{M} 2}-\theta_{\mathrm{M} 4}$ ). Selon Aubrey and Speer (1985) cités par Koutitonsky et al.(2007), plus le rapport $\mathrm{A}_{\mathrm{M} 4} / \mathrm{A}_{\mathrm{M} 2}$ est important, plus la distorsion de la marée est importante. Un rapport $\mathrm{A}_{\mathrm{M} 4} / \mathrm{A}_{\mathrm{M} 2}$ de l'ordre de 0.1 indique une faible distorsion tandis qu'un rapport $\mathrm{A}_{\mathrm{M} 4} / \mathrm{A}_{\mathrm{M} 2}>0.25$ indique une distorsion significative (Aubrey and Speer,1985). En étudiant ces rapports, Koutitonsky et al. (2007) ont constaté que le rapport des amplitudes $\left(\mathrm{A}_{\mathrm{M} 4} / \mathrm{A}_{\mathrm{M} 2}\right)$ est passé d'environ 0.1 dans la section aval de la lagune à près de 0.3 en amont, près de la digue (figures $3 \mathrm{c}$ et $\mathrm{d}$ ), ce qui est indicatif d'une importante distorsion de la marée à cet endroit. L'examen des phases relatives $(\Delta \theta)$ a aussi révélé que, dans le chenal principal, ces phases sont comprises entre 40 degrés et 70 degrés (figures $3 \mathrm{c}$ et d). Lorsque la phase relative est comprise entre 0 degrés et 180 degrés, cela confère à la lagune une dominance par le flux et que cette dominance est maximale à $\Delta \theta=90$ degrés (Koutitonsky et al., 2007).

\section{Courants dans la lagune de Oualidia}

La figure 4 présente les roses des courants mesurés dans la lagune de Oualidia aux stations C1 à C5 (figure 1) lors de la campagne de mesures effectuée par l'INRH entre juillet et août 2015. On constate que les courants observés à ces cinq stations présentent généralement des directions qui oscillent de 180 degrés entre le flot et le jusant et s'alignent généralement avec les axes des chenaux de la lagune. Les plus forts courants se manifestent durant les marées de vive-eau, respectivement de l'ordre de $1,25 \mathrm{~m} \cdot \mathrm{s}^{-1}$ à la station $\mathrm{C} 1 ; 1,05 \mathrm{~m} \cdot \mathrm{s}^{-1}$ à la station $\mathrm{C} 2 ; 0,40 \mathrm{~m} \cdot \mathrm{s}^{-1}$ à la station $\mathrm{C} 3 ; 0,35 \mathrm{~m} . \mathrm{s}^{-1}$ à la station $\mathrm{C} 4$. Ils diminuent d'intensité au fur et à mesure que l'on se dirige vers l'amont de la lagune. Une particularité a été, cependant, notée lors de cette campagne de mesures à la station C5 où les intensités du courant mesurées en marées de vives eaux étaient de l'ordre de $1 \mathrm{~m} . \mathrm{s}^{-1}$. Cette situation ne reflète pas réellement la courantologie dans cette partie amont de la lagune car le courantomètre (C5) a été installé volontairement à la digue aval artificielle, en vue d'estimer les volumes échangés entre la lagune et la partie située entre les digues aval et amont de la lagune (figure 1). Avec une brèche réduite de $10 \mathrm{~m} 2$ environ en 2005, cette digue aval artificielle va jouer le rôle d'un canal à dimension très réduite, intensifiant ainsi le courant à cette brèche. En périodes de marées de mortes-eaux, les intensités du courant observées aux cinq stations de la lagune diminuent et fluctuent généralement entre 0.1 à $0.2 \mathrm{~m} \cdot \mathrm{s}^{-1}$ (Hilmi et al., 2009).
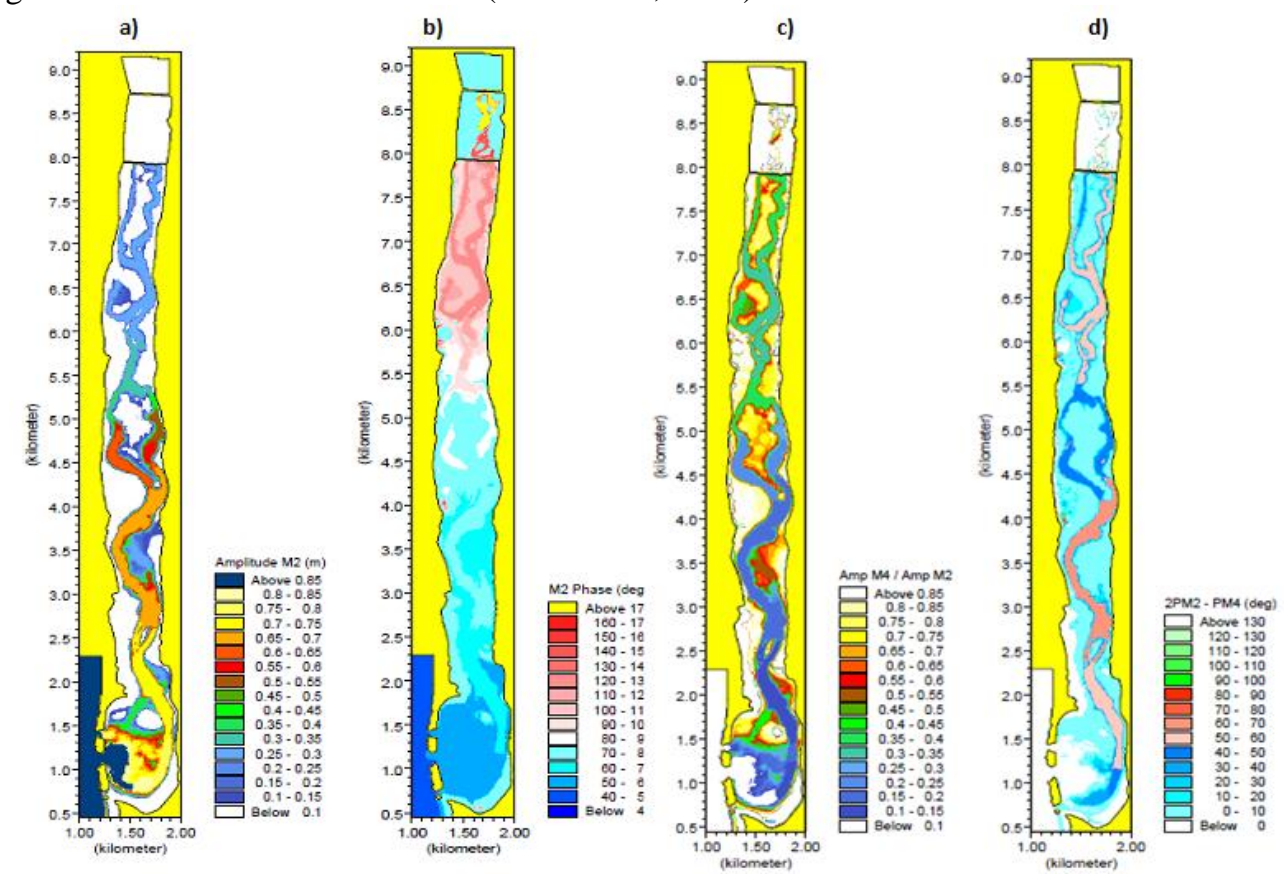

Figure 3:- a) Amplitude de l'harmonique M2 b) Phase de l'harmonique M2 c) Rapport d'amplitude M4/M2 et d) différence de phase 2M2-M4 (d'après Koutitonsky et al., 2007). 

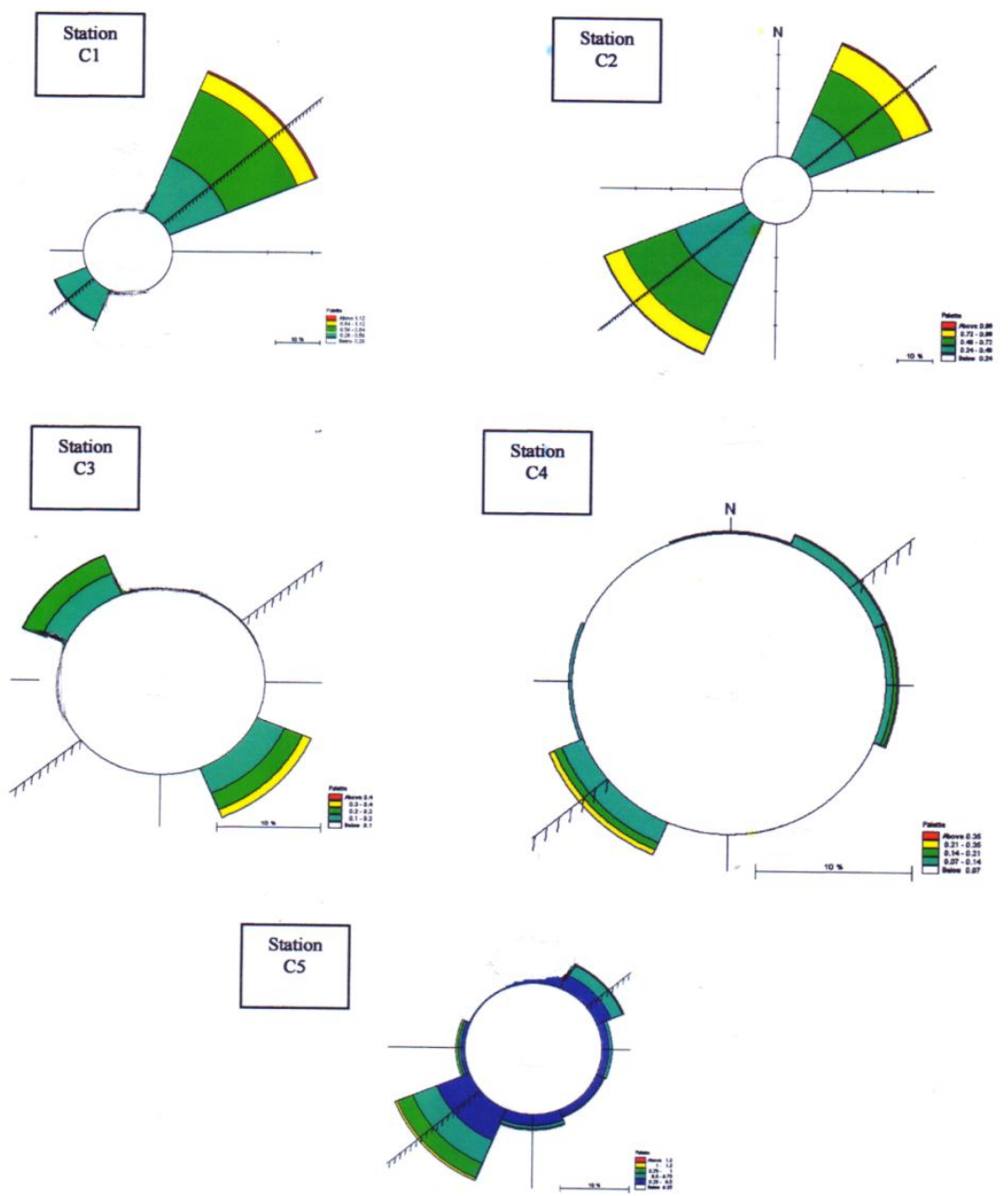

Figure 4:- Roses des courants observés dans la lagune de Oualidia aux stations de mesures $\mathrm{C} 1$ à $\mathrm{C} 5$ lors de la campagne effectuée par l'INRH entre juillet-août 2015 (d'après Hilmi et al., 2006, 2009).

En étudiant la répartition de l'énergie cinétique dans les basses et hautes fréquences ses séries temporelles du courant, la figure 5 met en évidence l'analyse spectrale des composantes U (est-ouest) et V (nord-sud) du courant à la station $\mathrm{C} 2$, par exemple. Cette figure confirme que l'énergie cinétique des courants est centrée à la période de la composante M2 et met aussi en évidence un niveau d'énergie cinétique relativement important proche de la période diurne $(24 \mathrm{~h})$. Cette énergie est normalement associée aux harmoniques diurnes de la marée K1 ou O1. Or, il a été clairement mis en évidence que la marée est de type semi-diurne dans la lagune de Oualidia (tableau 1 et figure 2) et que cette énergie ne pourrait être associée qu'aux oscillations inertielles qui se manifestent dans la zone côtière adjacente à la lagune de Oualidia (Hilmi et al., 2005b et 2009). La période inertielle $(\tau=2 \pi / \mathrm{f}$ ), où f est le paramètre de Coriolis à la latitude de la lagune, se situe aux alentours de 23h (Figure 5). Ces oscillations apparaissent généralement lors d'un changement brusque de la vitesse, de la direction et/ou du gradient spatial des vents à la surface de l'eau dont le forçage agirait sur le niveau d'eau et les courants dans la lagune à des «pseudo-périodes » situées entre 1,5 à $3 \mathrm{j}$ (Figure 5). 


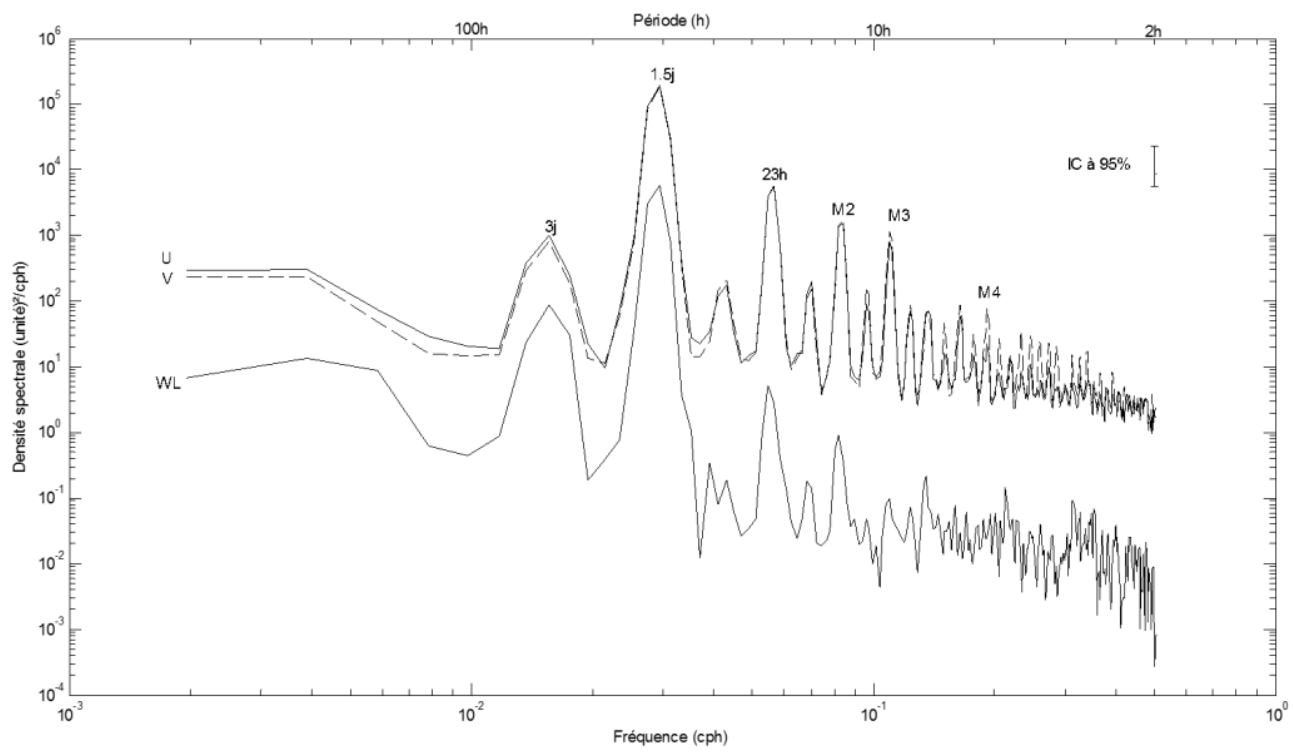

Figure 5:- Analyse spectrale des composantes U (Est_Ouest) (en traits pleins) et V (Nord-Sud) (en traits pointillés) du courant à la station C2 et du niveau d'eau à la station M1 par exemple (en traits pleins) dans la lagune de

Oualidia. L'intervalle de confiance à 95\% est calculé pour 8 degrés de liberté (Bendat et Piersol, 1986).

\section{Temps de Renouvellement:-}

Le temps de renouvellement des eaux de la lagune de Oualidia est un paramètre important dans la compréhension de son fonctionnement hydrodynamique et pour les activités aquacoles, d'autant plus que ce site abrite des parcs ostréicoles. La circulation et le renouvellement des eaux dans la lagune de Oualidia sont causés principalement par la marée à l'extérieur des passes d'entrée (Koutitonsky et al.,2006a, 2007). En utilisant la méthode du traceur dissout proposée par Koutitonsky et al. (2004), un temps de renouvellement local (TRL) et un temps de renouvellement intégral (TRI) sont mis en évidence. Ces temps de renouvellement furent étudiés pour la lagune de Oualidia par Hilmi et al (2005b), avec un temps de renouvellement local (TRL) variant entre 1 jour en aval près des passes d'entrée de la lagune et à environ $25 \mathrm{j}$ en amont de la lagune (à la digue aval artificielle) et un temps de renouvellement intégral (TRI) de 14 jours en moyenne. Les travaux actualisés et menés par Koutitonsky et al. (2007), sous la configuration de l'absence de la souille en amont de la lagune, ont permis de mettre en évidence que les eaux de la lagune de Oualidia sont renouvelées aux 15 jours environ (TRI) (figure 6a). S'agissant du temps de renouvellement local (TRL) des eaux de la lagune, ces auteurs ont mis en évidence que la partie aval de la lagune, près des passes d'entrée, est renouvelée par les eaux océaniques en l'espace d'un jour. À mesure que l'on se dirige vers l'amont, ce TRL augmente légèrement à 3-4 jours au niveau de la station «Palais Royal» (station C2) pour augmenter subitement à des temps de 10 à 20 jours dans la partie centrale de la lagune et de 20 à 30 jours dans la partie amont de la lagune (figure 6b). Le TRL à la digue aval artificielle serait ainsi de 30 jours environ (Koutitonsky et al., 2007) (figure 6b). En comparaison avec la lagune de Nador et sous la configuration de son ancienne passe d'entrée, le temps de renouvellement intégral (TRI) de cette lagune avant 2011 a été estimé à 80 jours environ (Hilmi et al., 2005c; Koutitonsky et al., 2006b). Suite à l'aménagement d'une nouvelle passe d'entrée dans la lagune de Nador qui est fonctionnelle depuis 2011, le nouveau temps de renouvellement est actuellement en cours d'étude. 


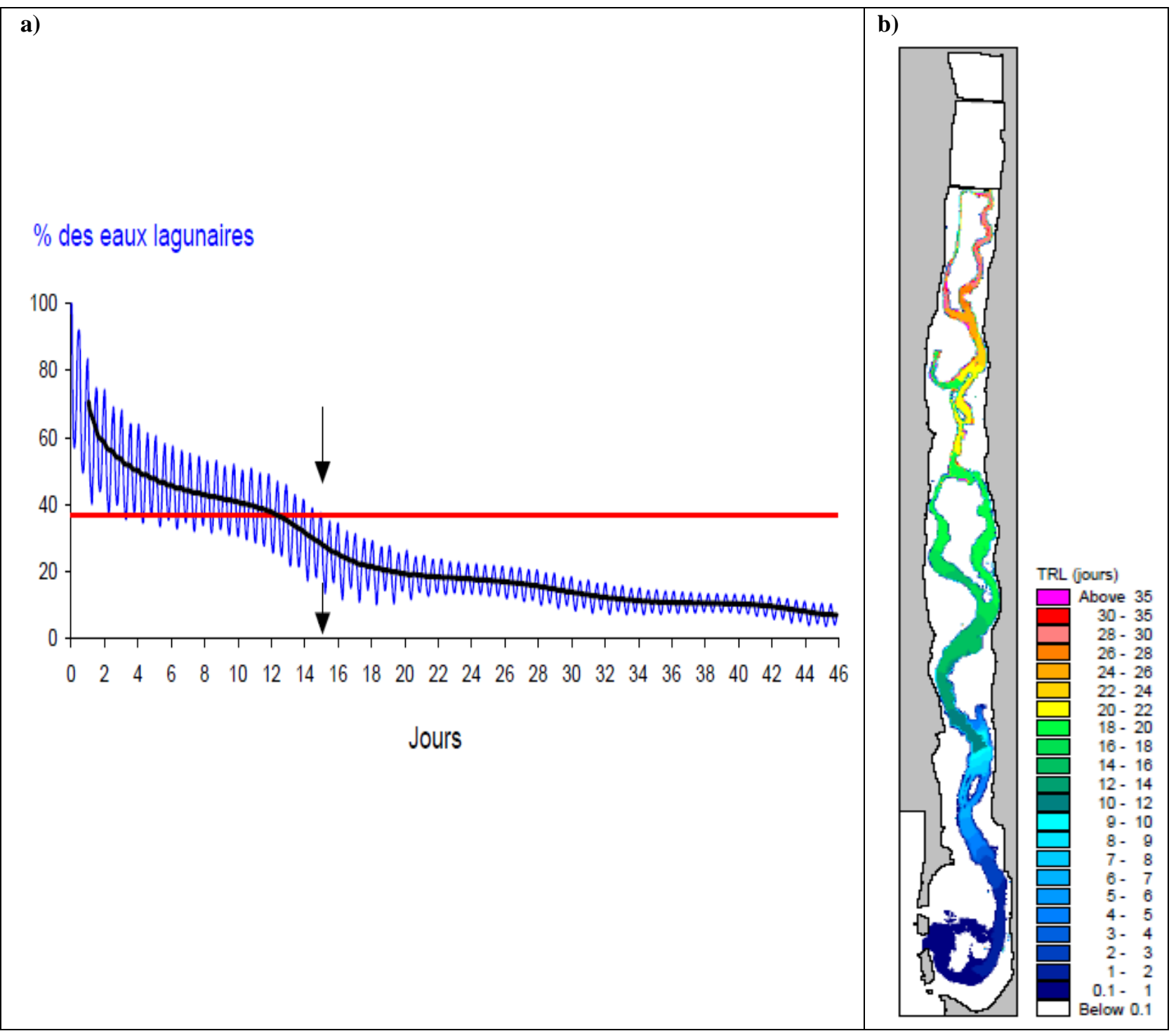

Figure 6:- a) temps de renouvellement intégral (TRI) et b) temps de renouvellement local (TRL) dans la lagune de Oualidia (d'après Koutitonsky et al., 2007).

\section{Température-Salinité:-}

Les diagrammes température-salinité permettent de caractériser les masses d'eau existantes, leurs origines et le type de mélange entre elles. Les observations de la température et de la salinité, mesurées aux stations courantologiques C1 à C5 (figure 1), vont être exploitées à cette étude. De manière à mieux interpréter les résultats en fonction des cycles de marée, les observations de la température et de la salinité, correspondant aux courants de flots, sont représentées par le symbole (*) et par le symbole (o) aux courants de jusants (Figure 7). D'une manière générale, l'aval de la lagune (stations $\mathrm{C} 1$ et $\mathrm{C} 2$ ) dénote une nette influence des eaux marines en provenance de l'océan Atlantique, compte tenu de leur proximité des deux passes de communication (figure 1). A la station $\mathrm{C} 1$, on note clairement la présence de deux masses d'eau bien distinctes: la première associée aux courants de flot où une salinité entre 35 et $36 \mathrm{psu}$ et une température variant entre $15^{\circ} \mathrm{C}$ et $18^{\circ} \mathrm{C}$ lui confèrent une densité d'environ $1026 \mathrm{~kg} \cdot \mathrm{m}^{-3}$ qui est typique de celle des eaux marines côtières. La deuxième masse d'eau, associée aux courants de jusant, présente des eaux moins denses $\left(\rho=1022 \mathrm{~kg} \cdot \mathrm{m}^{-3}\right)$, résultant du mélange avec des eaux relativement plus douces et chaudes en provenance des stations situées plus en amont de la lagune, en particulier à la station C2 (Figure 7). Cette dernière station se démarque par la présence de plusieurs sources d'eau douce situées le long de la rive continentale, à proximité du «Palais Royal» (Hilmi et al., 2006). Ces derniers auteurs ont, par ailleurs, recensé à marées basses 
lors de la campagne de mesures effectuées en 2005, de manière non exhaustive plus d'une cinquantaine de sources d'eaux douces réparties le long de la rive continentale de la lagune, du « Palais Royal » jusqu'à la digue amont de la lagune (figure 1). Plus on se déplace vers la partie intermédiaire de la lagune (stations C3 et C4) et vers l'amont de la lagune (station C5), plus l'on observe que les eaux subissent un mélange avec les résurgences d'eaux douces sous marines lors de leur parcours (Figure 7), créant ainsi un gradient longitudinal de densité. Plus en amont de la lagune et au niveau de la station C5 (digue aval artificielle), les températures fluctuent généralement entre $21^{\circ} \mathrm{C}$ et $32^{\circ} \mathrm{C}$ et les salinités entre 4 et 31 psu (Figure 7), ce qui dénote également la présence de nombreuses résurgences d'eau douces sous marines dans cette partie amont de la lagune et vers les marais salants (Hilmi et al, 2006, 2009). Ces résultats confirment aussi ceux de Rharbi et al. (2001 et 2003) où la typologie de la lagune, basée sur un suivi annuel hydrologique, a mis en évidence que la zone située en aval de la lagune est soumise à une nette influence marine. La zone centrale de la lagune est soumise à une double influence, marine et continentale et une zone confinée, située en amont de la lagune. Selon Rharbi et al. (2003), cette dernière zone correspond à la zone VI dans l'échelle de confinement établie par Guelorget et Perthuisot (1983).
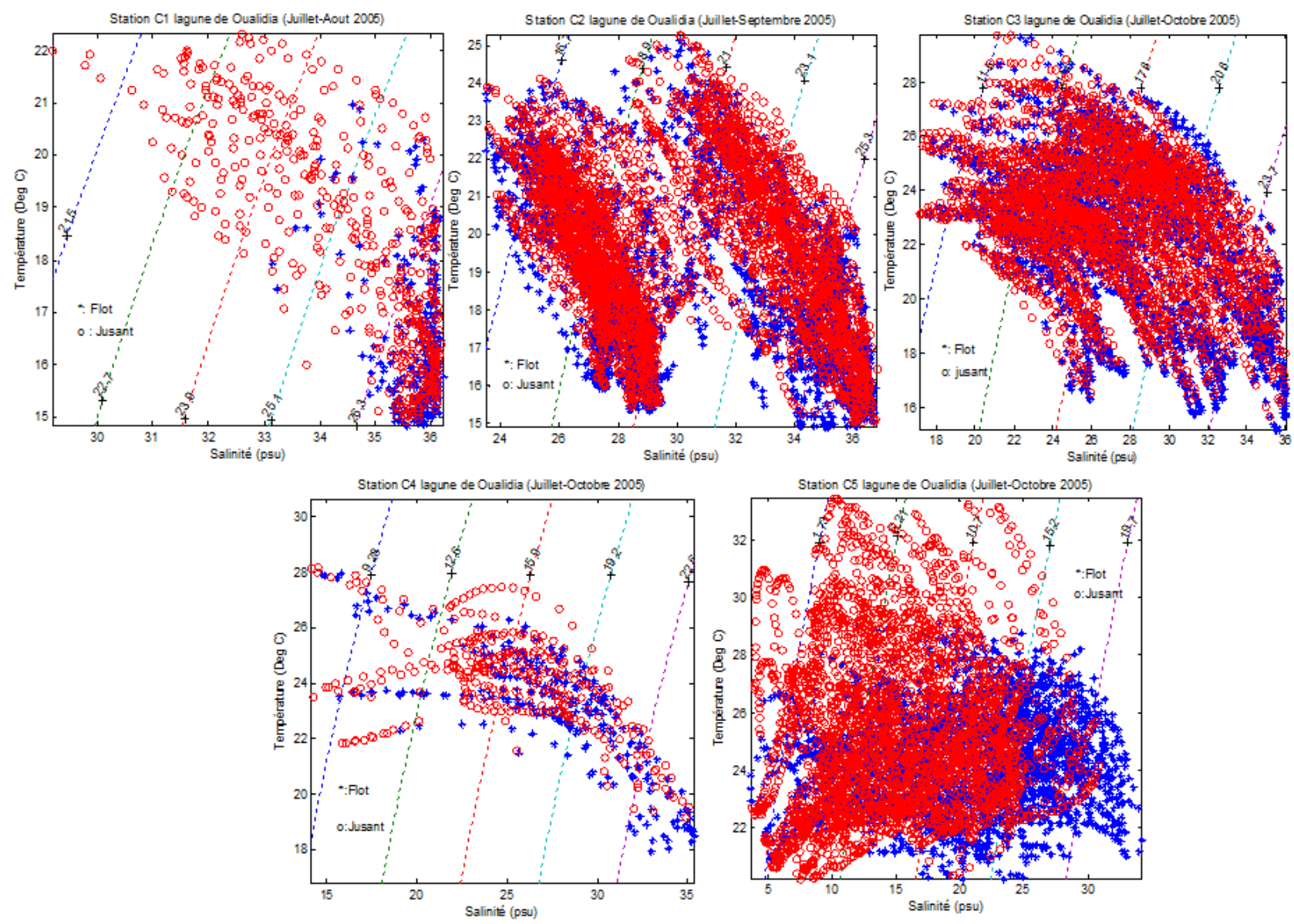

Figure 7:- Diagrammes Température-Salinité aux stations C1, C2, C3, C4 et C5 de la lagune de Oualidia durant la campagne de mesures de l'INRH en 2005 (d'après Hilmi et al, 2006, 2009).

\section{Conclusion:-}

Se basant sur de longues observations de marée, courant, température, salinité et de simulations par modélisation hydrodynamique 2D, cette étude, menée avant l'aménagement de la souille en 2011, a permis de mieux décrire et de comprendre les processus physiques agissant sur la circulation de la lagune de Oualidia. Le moteur de la circulation de cette lagune est la marée qui est semi-diurne (de type M2 de période 12,42h) dont l'amplitude est de $0.97 \mathrm{~m}$ à l'entrée de ses passes, asymétrique suite à la morphologie du chenal principal et des zones intertidales et perd de son amplitude au fur et à mesure qu'elle se propage au sein de la lagune. Son retard de phase est environ de $3 \mathrm{~h}$, en se propageant de l'extérieur de la lagune (passes d'entrée) à son extrémité amont (digue aval artificielle). 
Les courants observés dans la lagune sont essentiellement des courants de marée semi-diurnes dont les directions suivent celles du chenal principal et s'inversent de $180^{\circ}$ environ lors du renversement des marées. Ils sont très intenses $\left(>1 \mathrm{~m} \cdot \mathrm{s}^{-1}\right)$ en aval de la lagune à dominance de flot et moindres $\left(<0,5 \mathrm{~m} \cdot \mathrm{s}^{-1}\right)$ vers l'amont de la lagune. Les observations du courant dénotent la présence d'ondes de haute fréquence (de période $T \leq 25 \mathrm{~h}$ ) qui seraient attribuables aux oscillations inertielles du courant $(T \approx 23 \mathrm{~h})$ qui sont en rapport avec le vent dont le forçage agirait sur la lagune à des périodes variant entre 1,5 à 3j; à la marée semi-diurne M2 (de période 12.42h) ainsi qu'aux sous harmoniques de la marée semi-diurne $(\mathrm{M} 4, \mathrm{M} 3, \ldots)$ de périodes inférieures à $8 \mathrm{~h}$. Ces dernières, dues à la distorsion de l'onde de marée M2 le long du chenal principal et de sa morphologie et des ses faibles profondeurs dans la partie amont de la lagune, vont jouer un rôle prédominant dans l'asymétrie de la marée caractérisée par une dominance de flux. D'après Koutitonsky et al. (2007), la lagune demeure dominée par le flux avec un transport résiduel de boues vers l'amont de la lagune. En termes de renouvellement des eaux de la lagune, ces auteurs ont mis en évidence que le temps de renouvellement local (TRL) de la lagune de Oualidia varie entre 1 jour près des passes d'entrée de la lagune à 30 jours à sa digue aval artificielle et son temps de renouvellement intégral (TRI) est de 15 jours en moyenne. En termes d'hydrologie de la lagune, les températures et salinités observées sur la période d'étude indiquent une nette influence des eaux marines en aval de la lagune et une diminution de la salinité vers l'amont, due à la présence de nombreuses résurgences d'eaux douces dans la lagune en activités permanentes.

\section{Remerciements:-}

Cet article est dédié à la mémoire de feu Dr Jaouad Lakhdar Idrissi, chercheur à l'Institut National de Recherche Halieutique qui a beaucoup travaillé de son vivant dans la lagune de Oualidia. Un vibrant hommage est rendu au Professeur Vladimir Koutitonsky (Hydrosoft -Canada) qui a beaucoup contribué aux travaux scientifiques des lagunes de Oualidia et de Nador au Maroc depuis 2003. Les auteurs tiennent aussi à remercier le personnel de la station RSSL de l'INRH à Oualidia pour leur appui aux missions de terrain et des parcs ostréicoles à Oualidia notamment du Parc 07 ainsi que les évaluateurs externes de la revue.

\section{Références:-}

1. Aubrey, D. G., and Speer, P.E. (1985). A study of non-linear tidal propagation in shallow inlet/estuarine systems, Part 1: Observations. Estuarine, Coastal and Shelf Sciences, 21: 185-205.

2. Bendat, J-S., and Piersol, A.G. (1986). Random data: analysis and measurement procedures (Second Edition, Revised and Expanded). John Wiley \& Sons, New York, 1986, 566p.

3. Bennouna, A. (1999). Etude du phytoplancton du complexe lagunaire: Oualidia-Sidi Moussa. Thèse de 3ème Cycle, Université Chouaib Doukkali (Maroc), 149 p.

4. Bennouna, A., Berland, B., El Attar, J., and Assobhei, O. (2002). Eau colorée à Lingulodinium polyedrum (Stein) Dodge, dans une zone aquacole du littoral du Doukkala (Atlantique marocain). Oceanologica Acta, 25, 159-170.

5. Berraho, Ab. (1998). Ressources halieutiques et potentialités aquacoles du Maroc, Expo'98, Lisbonne, 82 p.

6. Bidet, J.C., and Carruesco, C. (1981). Étude sédimentologique de la lagune de Oualidia (Maroc). In: Proceedings International Symposium on coastal lagoons, SCOR/IABO/UNESCO, Oceanologica Acta, 29-37.

7. Blanton, J.O., Lin, G., and Elston, S.A. (2002). Tidal current asymmetry in shallow estuaries and tidal creeks. Continental Shelf Research, 22: $1731-1743$.

8. Bouchriti, N., El Marrakchi, A., and Fahim, A. (1982). The microbiological contamination of an oyster growing area in Morocco: The Oualidia lagoon. Hydroecol. Appl., 4 (2), 189-202.

9. Carruesco, C., (1989). Génèse et évolution à l'holocène de trois lagunes de la façade Atlantique: Moulay Bousselham, Oualidia (Maroc) et Arcachon (France), Thèse d'Etat, Université de Bodeaux I, Bordeaux, France, $485 \mathrm{p}$.

10. Chbicheb, A. (1996). Organisation biogéologique d'un bassin paralique: la lagune d'Oualidia (côte atlantique marocaine). Thèse de 3ème cycle, Univ. Nantes, 107p.

11. Cheggour, M., Chafik, A., Texier, H., Bouhallaoui, A., Rharbi, N., Boumezzough, A., and El Attar, J. (1999). Bioaccumulation de quelques éléments métalliques chez l'huître Crassostrea gigas (Thunberg) en élevage dans la lagune de Oualidia (Maroc) : Rôle des facteurs écologiques et biologiques. Haliotis, Vol. 28, 31-44.

12. Damsiri, Z., Elkalay, K, and Khalil, K. (2015). Modelling the biogeochemical cycle of the Oualidia lagoon (Atlantic, Morocco). International Journal of Advanced Research, Volume 3, Issue 2, 18-30, ISSN 2320-5407.

13. Dronkers, J. (1986). Tidal asymmetry and estuarine morphology. Netherlands, Journal of Sea Research. 20: 117-131.

14. El Attar, J. (1998). Contribution à la détermination de l'origine de la contamination fécale dans la lagune de 
Oulidia (Maroc) et étude de la contamination bactériologique de l'huître Crassostrea Gigas en conditions naturelles et expérimentales. Thèse de 3ème Cycle Univ. Chouaib Doukkali, 124 p.

15. El Khalidi, K., Zourarah, B., and Aajjane, A. (2011). Evolution récente de la morphologie de delta de flot et son effet sur la dynamique hydro-sédimentaire de la lagune de Oualidia (côte atlantique, Maroc): approche par photographie aérienne. Estudos do Quaternário, 7, APEQ, Braga, 2011, pp. 73-78.

16. Fahim, A. (1990). Etude de la pollution bactériologique de la lagune de Oualidia. Thèse de 3ème cycle Univ. Mohammed V, $213 \mathrm{p}$.

17. Fanul, E. A., Gomez, B. P., and Sanchez-Arevalo, I. R. (1997). A description of the tides in the Eastern North Atlantic. Prog. Oceanog., $40: 217-244$.

18. Ferre, M., and Ruhard, J.P. (1975). Les bassins des Abda-Doukkala et du Sahel de Azzemmour à Safi. Notes et Mémoires du Service Géologique, 23 : 261- 297

19. Friedrichs, C.T., and Madsen, O.S. (1992). Non-linear diffusion of the tidal signal in frictionally dominated embayments. Journal of Geophysical Research, 97:5637-5650.

20. GEME, Hydro-M, et In VIVO (2002). Diagnostic et études du confinement de la lagune de Oualidia- Phase 1 Etat Initial. Direction Provinciale de l'Equipement d'El Jadida, Ministère de l'Equipement et du Transport, Royaume du Maroc. Rapport définitif, 83 pp.

21. GEME, Hydro-M, et In VIVO (2003). Diagnostic et études du confinement de la lagune de Oualidia- Phase 2 Campagne de mesures. Direction Provinciale de l'Equipement d'El Jadida, Ministère de l'Equipement et du Transport, Royaume du Maroc. Rapport définitif, 60 pp.

22. GEME, Hydro-M, et In VIVO (2004). Diagnostic et études du confinement de la lagune de Oualidia- Phase 3 Préconisations techniques. Direction Provinciale de l'Equipement d'El Jadida, Ministère de l'Equipement et du Transport, Royaume du Maroc. Rapport définitif, 50 pp.

23. Guelorget, O., Perthuisot, J-P. (1983). Le domaine paralique. Expressions géologiques, biologiques et économiques du confinement., Trav. Lab. ENS, 16.

24. Godin, G. (1972). The Analysis of Tides. University of Toronto Press, Toronto, 264 p.

25. Hassou, N., Maanan, M., Hennani, M., Zourarah, B., and Assobhei, O. (2014). Spatial and temporal variation of faecal pollution indicators (Escherichia coli and faecal streptococci) and physico-chemical parameters at the Oualidia lagoon and its watershed (Morocco). International Journal of Current Microbiology and Applied Sciences, pp.675-694.

26. Hennani, M., Hassou, N., Aajjane, A. and Assobhei, O. (2014). Adjusting a primary survival model of Enteric Bacteria "E. coli" in coastal environment: Oualidia lagoon. Int.J.Curr.Microbiol.App.Sci (2014) 3(1): 127-139.

27. Hilmi, K., Orbi, A., Lakhdar, J.I. and Sarf, F. (2005a). Etude courantologique de la lagune de Oualidia (Maroc) en automne. Bull. Inst. Sci., Rabat, 26-27, 67-71.

28. Hilmi, K., Koutitonsky, V.G., Orbi, A., Lakhdar, J.I. and Chagdali, M. (2005b). Oualidia lagoon (Morocco): an estuary without a river. African Journal of Aquatic Sciences, 30-1, 1-10.

29. Hilmi, K., Koutitonsky, V.G., Orbi, A., and Chagdali, M. (2005c) Three dimensional water circulation and dispersion in Nador lagoon (Morocco). In: P. Lasserre, P. Viarioli and P. Campostrini Eds: Lagoons and Coastal Wetlands, in the Global Change Context: Impacts and Management Issues,Proceedings of the International Conference, Venice, 26-28 April 2004, ICAM Dossier N³,UNESCO.

30. Hilmi, K., Orbi, A. et Lakhdar, J.I. (2006). Campagne de mesures océanographiques menées durant l'été et l'automne 2005 dans la lagune de Oualidia. Rapport interne - Institut National de Recherche Halieutique, Royaume du Maroc, 20p.

31. Hilmi, K., Orbi, A. and Lakhdar, J.I. (2009). Hydrodynamisme de la lagune de Oualidia (Maroc) durant l'été et l'automne 2005. Bulletin de l'Institut Scientifique, Section Sciences de la Terre, Rabat, N’31.

32. Idardare, Z., Moukrim, A., Chiffoleau, J.-F., Ait Alla, A., Auger, D. and Rozuel, E. (2013). Evaluation de la contamination métallique dans deux lagunes marocaines: Khnifiss et Oualidia, Revue Marocaine des Sciences Agronomiques et Vétérinaires, Vol. 1, No 2 (2013).

33. INRH (2002). Ressources littorales: Etat de nos connaissances. Institut National de Recherche Halieutique, Document du Ministère des Pêches Maritimes, Royaume du Maroc, $110 \mathrm{p}$.

34. Kamara, A., Rharbi, N., Berraho, Ab., and Ramdani, M. (2005). Etude comparative du cycle sexuel de la palourde Ruditapes decussatus issue de trois milieux paraliques des côtes marocaines, Mar. Life 2005 - VOL.15 (1-2): 43-50.

35. Kamara, A., Rharbi., N., Ramdani, M. and Berraho, Ab. (2008). Recherches préliminaires au développement de l'élevage de la palourde européenne (Ruditapes decussatus L.) sur les côtes marocaines et au repeuplement des sites surexploités. Bull. Soc. zool. Fr., 133(1-3): 193-202. 
36. Koutitonsky, V.G., Guyondet, T., St. Hillaire, A., Courtenay, S. and Bohgen, A. (2004). Water renewal estimates for aquaculture developments in the Richibucto estuary, Canada. Estuaries, 27(5):839-850.

37. Koutitonsky, V.G., Orbi, A., Ouabi, M. et Ibrahimi I. (2006a). L'étude du comportement hydro-sédimentaire du système lagunaire Oualidia par la modélisation mathématique. Phase $1:$ Synthèse des données et simulations de la réfraction des houles. Direction des Ports et du Domaine Public Maritime, Ministère de l'Équipement et du Transport, Royaume du Maroc. $150 \mathrm{p}$.

38. Koutitonsky, V.G, Orbi, A., Hilmi, K., and Berraho, Ab. (2006b). Benefits of a proposed second inlet on water renewal in Nador lagoon, Morocco. The 1st International Conference: Environmental Change in lakes, lagoons \& wetlands of the southern Mediterranean region, 4-7 january 2006, Cairo, Egypt.

39. Koutitonsky, V.G., Ouabi, M. and Ibrahimi. I. (2007). L'étude du comportement hydrosédimentaire du système lagunaire Oualidia par la modélisation mathématique. Phase 2 : Modélisation hydro-sédimentaire de l'état actuel et de scenarios d'aménagement. Direction des Ports et du Domaine Public Maritime, Ministère de l'Équipement et du Transport, Royaume du Maroc. 204 p + Annexes .

40. Koutitonsky, V. G., Zyserman, J., and Zourarah, B. (2012). Étude par modèle mathématique de l'impact de l'ouverture de la digue amont et de l'enlèvement ou redistribution des sédiments de la sablière sur le comportement hydrosédimentaire de la lagune d'Oualidia. Mission 2: Modélisation hydro-sédimentaire de l'état actuel et des scénarios d'aménagement. Direction des Ports et du Domaine Publique Maritime, Ministère de l'Équipement et du Transport, Royaume du Maroc, 303 p. + Annexes.

41. Lakhdar, J.I., Orbi, A., Hilmi, K., Zidane, F. and Moncef, M. (2005). Modèles de température et de la qualité de l'eau appliqués à deux lagunes marocaines: Oualidia (Atlantqiue) et Nador (Méditerranée). Environmental Technology, Vol. 26, 713-720..

42. Maanan, M., Ruiz-Fernandez, A.C., Maanan, M., Fattal, P. and Zourarah, B. (2014). A long-term record of land use change impacts on sediments in Oualidia lagoon, Morocco. International Journal of Sediment Research, 29 (1), 1-10.

43. Orbi A., Hilmi, K., Larissi, J., Zidane, H., Zizah, S., El Moussaoui, N., Lakhdar, J.I., and Sarf, F. (1998). Hydrologie et hydrodynamique des côtes marocaines: milieux paraliques et zones côtières. Expo'98, Lisbonne, $68 \mathrm{pp}$.

44. Orbi, A., Hilmi, K., Lakhdar, J.I., and Zizah, S. (2008). Lagoon Ecosystem Study Trough Two Cases: Oualidia (Atlantic) and Nador (Mediterranean) - Morocco. Chapter In: Sustainable Use and Development of Watersheds - Part of the series NATO Science for Peace and Security Series pp 289-298.

45. Parker, B. (1991). The relative importance of the various non-linear mechanisms in a wide range of tidal interactions (Review). In: B. Parker [Ed.], Tidal Hydrodynamics, John Wiley \& Sons, NY, pp. 237-268.

46. Pawlowicz, R., Beardsley, B., and Lentz, S. (2002). Classical Tidal Harmonic Analysis Including Error Estimates in MATLAB using T_TIDE. Computers and Geosciences.

47. Pond, S., and Pickard, G.L. (1983). Introductory Dynamical Oceanography (2nd Edition). Pergamon Press, N.Y., $329 \mathrm{p}$.

48. Pugh, D.T. (1987). Tides, Surges and Mean Sea-Level. John Wiley \& Sons (Ed.), Chichester, 472 p.

49. Rharbi, N., Ramdani, M., Berraho, Ab. And Lakhdar, J.I. (2001). Caractéristiques hydrologiques et écologiques de la lagune de Oualidia, milieu paralique de la côte atlantique marocaine, Marine Life, 11, 3-9.

50. Rharbi, N., Ramdani, M. and Berraho, Ab. (2003). Elaboration d'une stratégie d'élevage de l'huître Crassostera gigas dans la lagune de Oualidia (Maroc) sur la base d'études des relations trophiques, Bull. Soc. Zool. Fr., $128,(1-2), 63-86$.

51. Shafee, M.S., and Sabatie, M.R. (1986). Croissance et mortalité des huîtres dans la lagune de Oualidia (Maroc). Aquaculture, 53, 201-214.

52. Sarf, F. (1999). Dynamique sédimentaire et état de pollution dans la lagune de Oualidia. Thèse de 3ème Cycle, Université Mohammed V (Maroc), $121 \mathrm{p}$.

53. Zourarah, B., Aajjane, A., El khalidi, K., Koutitonsky, V.G., and Zyserman, J. (2012). Étude par modèle mathématique de l'impact de l'ouverture de la digue amont et de l'enlèvement ou redistribution des sédiments de la sablière sur le comportement hydrosédimentaire de la lagune de Oualidia. Mission 1: Etude préliminaire. Direction des Ports et du Domaine Public Maritime, Ministère de l'Équipement et du Transport, Royaume du Maroc, 96 p. + Annexes. 